\title{
Escolhas e Ambiguidades: Um Estudo sobre o Conhecimento Comparativo*
}

\author{
Iansã Melo Ferreira ${ }^{\dagger}$, José Guilherme de Lara Resende ${ }^{\ddagger}$
}

\author{
Conteúdo: 1. Introdução; 2. Descrição e Aplicação do Experimento; 3. Resultados; \\ 4. Conclusão.
}

Neste artigo elaboramos dois experimentos e coletamos dados de 270 questionários aplicados a alunos da Universidade de Brasília, com o propósito de analisar a hipótese de ignorância comparada (CIH) de Fox e Tversky (1995). No primeiro experimento, testamos a CIH e, no segundo, investigamos os efeitos da comparação entre loterias com diferentes níveis de conhecimento sobre o processo de escolha individual. Os resultados obtidos não somente corroboram a hipótese de ignorância comparada, mas também aprofundam o seu entendimento, ao analisar o efeito da presença de mais de um parâmetro comparativo no processo decisório individual.

In this article, two experiments were conducted and data collected from 270 questionnaires applied to undergraduate students at the Universidade de Brasília, in order to study Fox and Tversky's comparative ignorance hypothesis (CIH) Fox e Tversky (1995). In the first experiment, we tested the $\mathrm{CIH}$, and in the second, we investigated the effects of comparison between lotteries with different degrees of competence in the individual decision making process. The results obtained not only corroborate the $\mathrm{CIH}$, but also deepen its comprehension, analyzing the effect of more than one parameter of comparison in the individual decision making process.

Palavras-chave: Ambiguidade, Hipótese de Ignorância Comparada, Efeitos de Comparação.

Códigos JEL: $\quad$ D03, D70, D81.

\footnotetext{
* Agradecemos a Rodrigo Peñalosa e Daniel Cajueiro pelos diversos comentários.

†Universidade de Brasília, Campus Universitário Darcy Ribeiro. Iansã Melo Ferreira agradece ao Conselho Nacional de Desenvolvimento Científico e Tecnológico ( $\mathrm{CNPq}$ ) pela bolsa de Mestrado que permitiu a realização deste trabalho. E-mail: iansa.mf@gmail.com

‡Universidade de Brasília, Campus Universitário Darcy Ribeiro. E-mail: jglresende@unb.br
} 


\section{INTRODUÇÃO}

Este artigo investiga o efeito comparação gerado por ordenamentos diferentes de questões com graus de familiaridade ou de conhecimento distintos. Para isso, elaboramos dois experimentos. 0 primeiro, de controle, replica o experimento "estudo 1" de Fox e Weber (2002), que testa a hipótese de ignorância comparada. O segundo testa como os efeitos de comparação se alteram quando apresentamos vários ordenamentos diferentes de conhecimento, onde cada ordenamento representa uma manipulação distinta do sentimento de competência individual. O objetivo de nosso estudo é aprofundar o entendimento da hipótese de ignorância comparada de Fox e Tversky (1995).

Os dois primeiros modelos de escolha individual sob incerteza, ainda amplamente usados, são a teoria da utilidade esperada (von Neumann e Morgentern, 1944) e a teoria da utilidade esperada subjetiva (Savage, 1954). A primeira se aplica a situações de risco e a segunda, a situações de incerteza ${ }^{1}$. Estas teorias foram e ainda são submetidas a grande escrutínio, e diversas falhas descritivas foram reveladas ao longo dos anos por meio de experimentos. As duas falhas mais famosas, conhecidas como o paradoxo de Allais (Allais, 1953) e o paradoxo de Ellsberg (Ellsberg, 1961), descrevem situações onde grande parte dos comportamentos observados não apenas contradizem as hipóteses destas teorias, mas contêm desvios sistemáticos dessas hipóteses. Esses dois paradoxos, além de outros encontrados na literatura, motivaram o surgimento de diversos estudos de cunho experimental (Camerer e Weber (1992) fazem uma excelente revisão desta literatura, Dellavigna (2009) revisa artigos com evidência experimental de desvios do comportamento modelado pela teoria de utilidade esperada) .

O paradoxo de Ellsberg baseia-se em experimentos simples, onde os agentes escolhem entre apostar em eventos incertos ou arriscados (Ellsberg, 1961, Becker e Brownson, 1964). No conhecido experimento de duas cores, os participantes devem escolher entre duas apostas, em que uma envolve a retirada de uma bola de determinada cor de uma urna com 100 bolas, nas quais 50 são brancas e 50 são pretas ("urna arriscada") e a outra envolve também a retirada de uma bola da mesma cor, também de uma urna com 100 bolas brancas e pretas, mas cuja proporção é desconhecida ("urna incerta"). Após selecionarem a aposta preferida (por exemplo, "ganhar $R \$ 20$ se uma bola branca é retirada da urna arriscada" versus "ganhar R\$ 20 se uma bola branca é retirada da urna incerta"), os participantes devem escolher também entre duas apostas similares envolvendo a bola de outra cor ("ganhar $R \$ 20$ se uma bola preta é retirada da urna arriscada" versus "ganhar $R \$ 20$ se uma bola preta é retirada da urna incerta"). Um número substancial de participantes prefere as apostas na urna arriscada para ambas as cores - preta e branca. Participantes que exibem esse padrão de escolhas evidenciam uma preferência pela urna cuja distribuição das cores das bolas é conhecida. Esse resultado constitui um paradoxo, já que escolher as duas apostas da urna arriscada viola os pressupostos da teoria de utilidade subjetiva esperada.

Embora os resultados desse experimento possam ser interpretados como uma simples aversão à incerteza knightiana, eles ilustram uma propensão de parte significativa dos participantes em apostarem em eventos sobre os quais se possui um nível mais elevado de conhecimento. Essa interpretação foi discutida por Heath e Tversky (1991), que propuseram a hipótese de competência, que postula que os indivíduos preferem participar de loterias em que se sintam mais competentes. Em uma série de experimentos, Heath e Tversky mostram que as pessoas podem ter uma preferência por incerteza, desde que possuam algum sentimento de competência sobre o assunto em questão. Logo, no caso do paradoxo de Ellsberg, os participantes fogem da urna incerta pois não possuem nenhum sentimento de competência em relação a ela.

A hipótese de competência procura então explicar como a existência de conhecimento prévio afeta decisões sob incerteza. Esta hipótese supõe que a propensão dos indivíduos a apostarem em um evento incerto depende não apenas da sua probabilidade estimada de ocorrência, mas também do nível de co-

\footnotetext{
${ }^{1}$ Knight (1921) diferenciou risco, incerteza mensurável por meio de uma distribuição de probabilidade, de incerteza (denominada também incerteza knightiana ou ambiguidade), a situação em que não é possível atribuir nenhuma distribuição de probabilidade para os resultados possíveis.
} 
nhecimento que o indivíduo possui (ou acredita possuir - o ponto fundamental é o indivíduo achar que é competente no assunto que delineia o evento, mesmo que não o seja, pois é o sentimento subjetivo de competência que induz o comportamento de propensão à incerteza) acerca do evento. Deste modo, para um mesmo valor de probabilidade julgada, o indivíduo pode preferir apostar em eventos sobre cujo contexto se considere mais conhecedor. Desse modo, volta-se à classificação de Keynes (1921), que definiu os conceitos de probabilidade julgada - a probabilidade atribuída, pelo indivíduo, a um evento; e peso das evidências - a credibilidade da fonte de informação. Assim, a hipótese de competência justifica uma preferência por incerteza knightiana, ou seja, "uma preferência por apostar em A em detrimento de B, embora B seja julgada ao menos tão provável quanto A" (Heath e Tversky, 1991, p. 09).

Uma justificativa psicológica para tal comportamento de propensão à incerteza é se, por um lado, um indivíduo que possui expertise em determinado assunto pode se sentir mais envergonhado por uma previsão errada sobre este, por outro, o expert costuma possuir argumentos que balizam as suas escolhas, amenizando possíveis julgamentos derivados de seu erro. Heath e Tversky ressaltam esta racionalização (1991, p. 07):

"There are both cognitive and motivational explanations for the competence hypothesis. People may have learned from lifelong experience that they generally do better in situations they understand than in situations they have less knowledge(...)"

Fox e Tversky (1995) desenvolveram a hipótese de ignorância comparada (CIH), um aprimoramento da hipótese de competência de Heath e Tversky. Os dois autores argumentaram que, no momento de suas escolhas, o indivíduo não considera suas dotações de conhecimentos ou habilidades isoladamente, mas sim de maneira relativa.

Em particular, Fox e Tversky modificaram o experimento de Ellsberg, fazendo com que os participantes não mais escolhessem entre apostas diferentes, mas sim reportassem quanto estariam dispostos a pagar por cada aposta apresentada (o equivalentes de certeza da aposta). Os autores dividiram os participantes em dois grupos, um comparativo e outro não-comparativo. Os participantes do grupo comparativo reportavam os equivalentes de certeza das apostas para ambas as urnas. Já os participantes do grupo não-comparativo foram subdivididos em dois subgrupos, onde o primeiro reportou os equivalentes de certeza das duas apostas referentes à urna arriscada e o segundo subgrupo reportou os equivalentes de certeza das duas apostas referentes à urna incerta. A Tabela 1 apresenta os resultados obtidos por Fox e Tversky.

Tabela 1: de Fox e Tversky (1995)

\begin{tabular}{ccc}
\hline & Clear Bet & Vague Bet \\
\hline Comparative & $\$ 24,34$ & $\$ 14,85$ \\
& $(2,21) N=67$ & $(1,80) N=67$ \\
Noncomparative & $\$ 17,94$ & $\$ 18,42$ \\
& $(2,50) N=35$ & $(2,87) N=39$ \\
\hline
\end{tabular}

Esta Tabela 1 mostra que a aversão à incerteza knightiana desaparece no ambiente não-comparativo: a diferença entre a soma dos valores dos equivalentes de certeza entre as apostas da urna com risco (coluna "clear bet") e as apostas da urna com incerteza (coluna "vague bet"), no caso não-comparativo (linha "Noncomparative"), são desprezíveis. Chow e Sarin (2001) replicaram o experimento de Fox e Tversky e apesar de não encontrarem que a aversão à incerteza knightiana desapareça por completo na situação não-comparativa, os resultados obtidos mostraram que ela diminui consideravelmente.

Segundo a $\mathrm{CIH}$, as escolhas individuais em um ambiente com incerteza podem ser afetadas por efeitos de comparação, que podem tomar várias formas. Por exemplo, no experimento de Ellsberg, o 
efeito é causado pela comparação entre as duas urnas, que possuem níveis diferentes de conhecimento. Este efeito adiciona um componente volátil ao comportamento decisório individual, já que os agentes tomam suas decisões com base em um sentimento de competência comparativo e, como tal, sujeito à manipulação. Outros estudos investigaram a hipótese de ignorância comparada (Fox e Tversky, 1998, Fox e Weber, 2002, Chow e Sarin, 2001), todos confirmando a existência de efeitos de comparação nas escolhas individuais. Esses artigos evidenciam que existe um forte efeito de comparações sobre as escolhas realizadas e avançam a análise proposta por Ellsberg.

Experimentos baseados em urnas foram criticados ao longo dos anos (ver, por exemplo, Heath e Tversky (1991) e Fox e Tversky (1995)), com base no fato de que as decisões tomadas pelos indivíduos nesses experimentos são desligadas do cotidiano e, portanto, não refletem o comportamento que esses agentes teriam ao confrontar-se com problemas reais. Assim, novos experimentos foram elaborados, com loterias que contemplassem eventos reais ao invés de urnas hipotéticas. Essas loterias apresentam situações onde, em contextos comparativos e não-comparativos, os agentes são questionados acerca de temas sobre os quais possuam diferentes graus de conhecimento, permitindo captar o impacto de efeitos de competência e de comparação sobre as decisões individuais.

Esses estudos concluem que o sentimento de competência (ou ignorância) comparada - em oposição à competência (ou ignorância) absoluta - é uma variável chave no processo de escolha individual. No momento de tomar uma decisão, as pessoas usam não o seu nível de conhecimento absoluto, mas sim o seu nível de conhecimento relativo, que pode ser relativo à existência de outros agentes com maior conhecimento sobre o evento, relativo à existência de eventos que lhes pareçam mais familiares, ou relativo a qualquer outro fator que possa alterar a perpcepção de conhecimento do indivíduo.

Em particular, Fox e Weber (2002, p. 485) sugerem a existência de efeitos de ordenação sobre escolhas realizadas em um ambiente ambíguo:

"When a survey contains bets that vary in their familiarity, source preference will be more pronounced for later sources than for the first source evaluated."

Portanto, o ordenamento das alternativas com o qual se depara o indivíduo pode afetar as escolhas realizadas. De acordo com a $\mathrm{CIH}$, a comparação dos níveis de conhecimento que os indivíduos possuem com relação a diferentes eventos faz com que eles atribuam valores distintos a uma mesma loteria. Em um ambiente comparativo, a ordenação das escolhas pode induzir, nos indivíduos, um determinado nível de percepção sobre o próprio conhecimento, fazendo-os redimensionar suas crenças e influenciando suas escolhas.

Em particular, o experimento 1 de Fox e Weber (2002, study 1, p. 481), com 110 estudantes da Willamette University, em Salem, Estados Unidos, analisou efeitos de comparação em um contexto diferente do da urna de Ellsberg. Neste experimento, eram apresentadadas duas escolhas ao participante, onde cada escolha embutia um nível distinto de familiaridade. 0 experimento visava a testar se o ordenamento dessas escolhas influencia o processo decisório individual.

Os resultados obtidos pelos autores indicaram que uma escolha sobre um tema muito familiar antes de uma sobre um tema de familiaridade moderada induz os indivíduos a sub-avaliarem o próprio conhecimento a respeito do tema concernente à segunda escolha. Porém, uma escolha sobre um tema pouco familiar antes de uma escolha sobre um tema de familiaridade moderada induz os indivíduos a se considerarem mais conhecedores do tema concernente à segunda escolha. Deste modo, a ordem das escolhas modificou o comportamento perante a incerteza, induzindo, até mesmo, aversão ou preferência por incerteza knightiana.

Mas, em que extensão ordenações diferentes de um mesmo conjunto de perguntas levam a diferentes resultados? Se as três perguntas com diferentes níveis de conhecimento são feitas, apenas em ordenações distintas - 1) mais familiar, 2) menos familiar, 3) moderadamente familiar e 1) menos familiar, 2) mais familiar, 3) moderadamente familiar, qual o efeito sobre o comportamento decisório individual? Teria a primeira pergunta algum efeito sobre a segunda e esta sobre a terceira? Haveria um 
"cancelamento" dos efeitos comparativos de modo a anular os vieses? Estas são as principais questões que motivam o artigo. Os experimentos que se seguem foram desenhados com o propósito de elucidar estas questões.

$O$ resto do artigo é organizado da seguinte maneira. A Seção 2 descreve o experimento e a sua aplicação. A Seção 3 apresenta a análise dos dados e discute os resultados obtidos. A Seção 4 conclui.

\section{DESCRIÇÃO E APLICAÇÃO DO EXPERIMENTO}

O presente estudo realizou dois experimentos para investigar o comportamento decisório e o funcionamento da memória comparativa individual. O primeiro serve como experimento de controle, ao replicar o "estudo 1" realizado por Fox e Weber (2002). O segundo estende o primeiro, acrescentando mais uma pergunta, de modo a estabelecer um parâmetro adicional de competência. Esta extensão permite uma análise mais abrangente do fluxo de indução comparativa sobre as escolhas dos agentes. A seguir apresentaremos a ideia e o formato dos experimentos e, depois, os procedimentos de aplicação e os resultados alcançados.

O ponto de partida para a elaboração dos experimentos foi o "estudo 1" de Fox e Weber (2002, study 1 , p. 481). Foram escolhidos eventos os mais semelhantes possíveis, que diferissem apenas no nível de competência do público respondente. Assim, escolhemos três cidades cujas amplitudes térmicas e temperaturas médias em determinada data fossem semelhantes, mas de modo que os graus de conhecimento dos participantes a respeito dessas cidades fossem distintos.

Os questionários foram aplicados em Brasília ao longo do mês de abril de 2009. A data de referência selecionada foi 10 de maio do mesmo ano. As cidades escolhidas foram as cidades brasileiras de Brasília-DF e Belo Horizonte-MG e a cidade egípcia de Zagazig, na Província de Sharqiyyah. Na média, a probabilidade de ocorrência de determinada temperatura nestas cidades é a mesma para o mês de maio. Consideramos que um morador de Brasília sentir-se-ia mais competente a respeito da primeira, moderadamente competente a respeito da segunda e pouco ou nada competente a respeito da terceira.

Assim, os eventos utilizados foram:

1. Temperatura na cidade de Brasília (Tbsb) - evento de alta familiaridade;

2. Temperatura na cidade de Belo Horizonte (Tbh) - evento de familiaridade moderada;

3. Temperatura na cidade de Zagazig (Tzz) - evento de baixa familiaridade.

O primeiro experimento (experimento 1), replicou o estudo 1, de Fox e Weber (2002), visando a testar a hipótese de ignorância comparada por meio da aplicação de dois questionários distintos, abordando dois eventos cada. Os dois questionários diferiam apenas em relação à indução do efeito comparativo. As escolhas foram apresentadas da seguinte maneira:

Tabela 2: Experimento 1

\begin{tabular}{lcc}
\hline & Questionário 1A & Questionário 1B \\
\hline Primeiro Evento & Tzz & Tbsb \\
Segundo Evento & Tbh & Tbh \\
\hline
\end{tabular}

Para ambos os questionários, as perguntas concernentes ao experimento foram escritas com base na estrutura frasal de Fox e Weber e comum nesta literatura. A pergunta foi estruturada do seguinte modo:

"Imagine que lhe foi oferecida uma aposta a qual pagará $\mathrm{R} \$ 100$ se a mais alta temperatura da tarde na cidade de [CIDADE, ESTADO OU PROVÍNCIA], for IGUAL OU MAIOR QUE (OU 
MENOR QUE) $25^{\circ} \mathrm{C}$ no próximo dia 10 de maio. Qual o máximo que você pagaria para entrar nessa aposta?

O máximo que eu pagaria para entrar nessa aposta seria: RS

Para cada cidade, foram realizadas duas perguntas complementares ("temperatura igual ou maior que" e "temperatura menor que"), de modo que qualquer crença a respeito das temperaturas pudesse ser expressa. Para cada novo par de perguntas, apenas o nome da cidade foi modificado, de modo a potencializar o efeito comparativo do experimento.

Após precificar as loterias apresentadas, o participante classificou o seu conhecimento a respeito dos eventos em uma escala baseada em de Lara Resende e Wu (2010) que vai de 1 - nenhum conhecimento - até 7 - excelente conhecimento. $O$ uso da escala permite analisar a adequação da escolha dos eventos e, também, testar se o ordenamento dos eventos afeta o grau de competência reportado pelos participantes. Além disso, a inserção desta escala no experimento nos permite excluir da análise indivíduos cujas respostas não se encaixem na ordenação de conhecimento presumida.

O segundo experimento (experimento 2) assemelha-se ao primeiro. A estrutura frasal, a realização de perguntas complementares e a escala para determinação dos níveis de conhecimento foram mantidas para os questionários deste experimento, que se diferenciaram dos questionários do primeiro por abordar não apenas dois, mas os três eventos acima citados, em ordenações distintas, mantendo-se sempre Tbh como última loteria a ser analisada pelos participantes. As perguntas no segundo experimento foram apresentadas conforme segue:

Tabela 3: Experimento 2

\begin{tabular}{ccc}
\hline & Questionário 2A & Questionário 2B \\
\hline Primeiro Evento & Tzz & Tbsb \\
Segundo Evento & Tbsb & Tzz \\
Terceiro Evento & Tbh & Tbh \\
\hline
\end{tabular}

O objetivo do experimento 2 foi investigar o fluxo indutivo da percepção de ignorância comparada. Ou seja, se a "memória comparativa" dos indivíduos gera um efeito sobre a percepção de conhecimento; ou se este efeito se prolonga, afetando perguntas "mais distantes"; ou ainda, se os efeitos derivados dos diferentes parâmetros se cancelam ao longo do caminho.

Seguindo a estrutura de Fox e Weber (2002), para cada par de perguntas complementares, foram somados os valores atribuídos pelos respondentes. Por exemplo, somamos, para cada participante, o valor por ele atribuído à loteria que premiava $\mathrm{Tbsb} \geq 25^{\circ}$, ao valor atribuído à loteria que premiava $\mathrm{Tbsb}<25^{\circ}$. Desse modo, o valor atribuído pelo indivíduo não é viesado pela sua crença em uma temperatura maior ou menor que a de referência. As análises foram realizadas com base nos valores médios dessas somas, procurando refletir o sentimento dos agentes a respeito da relação de seus níveis de competência.

Os dados coletados foram utilizados para realizar as seguintes análises. Primeiro, estudamos o efeito da memória comparativa dos indivíduos, utilizando a diferença entre os valores atribuídos às loterias que abordam Tbh no experimento 1 (CIH testada por Fox e Weber).

Em seguida, supondo que os participantes se sentiam mais seguros em classificar o próprio conhecimento a respeito dos eventos Tbsb (conhecimento alto) e Tzz (conhecimento baixo) do que se sentem com relação a Tbh, analisamos os dados obtidos com os questionários $2 \mathrm{~A}$ e $2 \mathrm{~B}$, comparando as respostas obtidas para Tbsb e Tzz quando apresentadas em primeiro ou em segundo lugar. Desse modo, examinamos o tamanho do efeito indutivo sobre esses eventos, assumindo-o inferior ao efeito gerado por eles sobre Tbh, uma vez que o participante define melhor o seu conhecimento sobre aqueles. Se nesses casos não é encontrada diferença significativa nos valores atribuídos a uma pergunta - digamos a de 
maior familiaridade - quando esta é feita antes ou depois da outra - digamos a de menor familiaridade -, isso indica que o efeito comparação é desprezível.

O segundo experimento permitiu também analisar se há diferença entre os vieses gerados pelas duas primeiras perguntas sobre a terceira. Para isso, primeiro comparamos as respostas dos questionários tipo $2 \mathrm{~A}$ e $2 \mathrm{~B}$, com relação à última pergunta - de familiaridade moderada - para verificar se existe uma diferença entre os valores atribuídos e, nesse caso, qual o efeito predominante. Além disso, comparamos também os resultados obtidos nos questionários com duas e com três perguntas, a fim de observar se a inserção de mais um parâmetro comparativo (transformando um questionário tipo 1 em um questionário tipo 2) afeta a percepção de competência do indivíduo a respeito do evento de familiaridade moderada.

Os questionários foram aplicados a 270 estudantes da Universidade de Brasília, em três turmas da disciplina "Introdução à Economia". As turmas contemplavam grupos heterogêneos, com alunos de diferentes cursos das áreas de exatas, humanas e até mesmo saúde. Dentre os respondentes, 14 foram excluídos por violar dominância estocástica e 20 por apresentarem ordenações de conhecimento que contradisseram a hipótese assumida - a temperatura em Brasília representar um evento muito familiar; a temperatura em Belo Horizonte, um evento moderadamente familiar e a temperatura em Zagazig, um evento pouco familiar.

Os quatro modelos de questionários foram aplicados simultaneamente em cada uma das turmas, onde foram distribuídos de forma aleatória. A aplicação se deu entre 10 e 20 dias antes da data de referência das loterias (10 de maio de 2009). Aproximadamente metade dos estudantes respondeu ao primeiro experimento, e o restante ao segundo. Em ambos os casos, os estudantes foram convidados a participar durante o horário normal de aula. Para reduzir contra-incentivos, a aplicação dos questionários aconteceu entre o início e o meio da aula, evitando que "a pressa em responder para então sair de sala" distorcesse os resultados. Além disso, seguindo Fox e Weber (2002), a aplicação do experimento não teve contrapartida de nota, créditos acadêmicos, ou fiduciária. Assumiu-se então que os indivíduos expostos a situações hipotéticas conseguem reproduzir as decisões que tomariam em situações reais ${ }^{2}$.

\section{RESULTADOS}

Vamos apresentar primeiro as avaliações de competência para depois apresentarmos os resultados referentes às escolhas realizadas. A Tabela 4 apresenta a média das respostas à pergunta de competência. Ela confirma a classificação assumida pelos autores, como esperado, onde os participantes declaram ser mais competentes a respeito da temperatura em Brasília do que em Belo Horizonte e mais competentes a respeito desta do que da temperatura de Zagazig.

A Tabela 4 evidencia como o efeito comparação ocorre a partir de uma percepção subjetiva da competência individual. Quando foi pedido aos participantes que avaliassem o seu conhecimento do evento Tbh conjuntamente com o evento $\mathrm{Tzz}$, a média dada para $\mathrm{Tbh}$ foi 4,38, superior à média 2,33 atribuída quando o evento Tbh foi avaliado em conjunto com o evento Tbsb. Por outro lado, quando analisado em conjunto com Tbsb e Tzz, Tbh recebeu valores próximos em ambos os questionários (3,48 e 3,61), valores estes intermediários aos obtidos no experimento 1 .

Vamos agora apresentar os resultados referentes às escolhas realizadas, que permitem analisar a hipótese da ignorância comparada. Os resultados do experimento 1 mostram uma diferença de mais de R\$ 10,00 entre a média do valor atribuído a Tbh nos dois tipos de questionários, 1A e 1B (ver Tabela 5). Encontramos aqui o mesmo resultado apresentado por Fox e Weber, corroborando a hipótese de ignorância comparada. Observamos ainda a atribuição de valores bastante distintos para as loterias iniciais

\footnotetext{
${ }^{2}$ A questão de incentivos financeiros em experimentos é controversa. Camerer e Hogarth (1999) revisam 74 artigos e concluem que a presença de incentivos monetários em experimentos não é clara - podendo ajudar em certos tipos de experimentos e atrapalhar em outros. Porém, Hertwig e Ortmann (2001), após analisar também uma série de estudos, concluem que na maioria deles, incentivos financeiros fazem diferença.
} 
Tabela 4: Grau de Competência Médio

\begin{tabular}{lccc}
\hline & \multicolumn{3}{c}{ Eventos } \\
\hline & Tbsb & Tbh & Tzz \\
\cline { 2 - 4 } Questionário 1A & \multicolumn{3}{c}{ Experimento 1} \\
\cline { 2 - 4 } Questionário 1B & 4,82 & 2,33 & -14 \\
& \multicolumn{3}{c}{ Experimento 2} \\
\cline { 2 - 4 } Questionário 2A & 5,16 & 3,48 & 1,21 \\
Questionário 2B & 5,26 & 3,61 & 1,06 \\
\hline Média & 5,08 & 3,45 & 1,14 \\
\hline
\end{tabular}

- Tzz no questionário 1A e Tbsb no questionário 1B. Neste caso, onde há ausência de parâmetros de comparação, obtivemos resultado semelhante ao encontrado por Chow e Sarin (2001), que concluíram que em um ambiente não-comparativo, a ambiguidade se torna um parâmetro secundário, passando a ser subavaliada e até mesmo negligenciada.

Tabela 5: Resultados dos Experimentos 1 e 2

\begin{tabular}{lcc}
\hline & \multicolumn{2}{c}{ Experimento 1 - Dois Eventos } \\
& Questionário 1A $(n=60)$ & Questionário 1B $(n=60)$ \\
\cline { 2 - 3 } Primeira Pergunta & Zagzig: R\$ 38,79 & Brasília: $\mathrm{R} \$ 58,47$ \\
Segunda Pergunta & Belo Horizonte: $\mathrm{R} \$ 54,08$ & Belo Horizonte: $\mathrm{R} \$ 42,38$ \\
\hline & \multicolumn{2}{c}{ Experimento 2 - Três Eventos } \\
& Questionário 2A $(n=58)$ & Questionário 2B $(n=54)$ \\
\cline { 2 - 3 } Primeira Pergunta & Zagzig: $\mathrm{R} \$ 45,16$ & Brasília: $\mathrm{R} \$ 65,73$ \\
Segunda Pergunta & Brasília: $\mathrm{R} \$ 68,29$ & Zagzig: $\mathrm{R} \$ 26,85$ \\
Terceira Pergunta & Belo Horizonte: $\mathrm{R} \$ 58,17$ & Belo Horizonte: $\mathrm{R} \$ 50,00$ \\
\hline
\end{tabular}

Ao analisar o efeito do ordenamento para os eventos Tbsb e Tzz no experimento 2, verificamos novamente a ocorrência do viés comparativo. De fato, o efeito sobre a percepção de conhecimento acerca de Tzz, medida pela diferença entre os valores atribuídos para Tzz nos questionários $2 \mathrm{~A}$ e $2 \mathrm{~B}$, é no valor de $\mathrm{R} \$ 18,31$. Por outro lado, o efeito comparativo de Tzz sobre Tbsb mostrou-se bastante reduzido, elevando o preço médio atribuído a esta loteria em não mais que $\mathrm{R} \$ 2,56$. Ao observar essas diferenças, vemos que o impacto comparativo maior dá-se sobre Tzz, seguido por Tbh e, finalmente, Tbsb. Uma possível justificativa para esse comportamento pode ser a maior segurança dos respondentes em relação ao evento, quanto maior seu grau de familiaridade. Ou seja, quanto mais alto o grau de familiaridade do indivíduo acerca de determinado evento, menor seria o poder de distorção da percepção de conhecimento pela provisão de parâmetros comparativos.

Analisando os valores atribuídos a Tzz e Tbsb, quando posicionados como segunda pergunta, no experimento 2, é possível constatar que, como postulado pela $\mathrm{CIH}$, em um ambiente comparativo, o sen- 
timento de ignorância (ou competência) é amplificado. De fato, a diferença entre os valores atribuídos mais do que dobra com relação à diferença de $R \$ 20,00$ observada entre as mesmas loterias na ausência do parâmetro comparativo (quando apresentadas como primeira pergunta).

Outro resultado inferido a partir do segundo experimento foi que a memória comparativa parece acompanhar o primeiro parâmetro apresentado e não necessariamente o mais próximo. Em outras palavras, quando acrescentamos uma terceira escolha em um questionário com duas escolhas, transformando-o em um questionário do tipo 2 , o sentimento comparativo que predomina sobre a última escolha (Tbh) é aquele induzido pela primeira.

Vamos então comparar os questionários cuja primeira pergunta coincide, i.e., $1 \mathrm{~A}$ e $2 \mathrm{~A}$; $1 \mathrm{~B}$ e $2 \mathrm{~B}$. Ao confrontar o impacto da primeira pergunta sobre a última, medido pela diferença entre os valores atribuídos às duas, nesses dois pares de questionários, temos que, para os questionários iniciados pelo evento Tzz (tipo A), $\mid \mathrm{Tzz}$ - Tbh| é pouco maior para o experimento com apenas duas perguntas. Da mesma forma, para os questionários iniciados pelo evento Tbsb (tipo B), |Tbsb - Tbh| é também pouco maior para o experimento com duas perguntas, indicando que a inserção de mais um parâmetro comparativo reduz, mesmo que timidamente, o impacto gerado pelo primeiro parâmetro estabelecido.

Os resultados inferidos com base unicamente nos valores médios atribuídos às loterias podem não ser estatisticamente válidos. Para aferir a significância estatística desses resultados, utilizamos o teste U de Mann-Whitney, destinado à comparação de grupos independentes, cujas observações sejam ordenáveis. De acordo com os resultados deste teste, corroboramos a análise feita acima sobre a hipótese de ignorância comparada, com $\operatorname{Tbh}(1 \mathrm{~A})-\operatorname{Tbh}(1 \mathrm{~B})>0$, para $t=5 \%$ e valor $p=0,046$ e, por outro lado, $\operatorname{Tzz}(1 \mathrm{~A})-\mathrm{Tbsb}(1 \mathrm{~B})<0(t=5 \%$ e $p=0,000)$. Além disso, verificamos um efeito indutivo sobre $\mathrm{Tzz}$, ocasionado por Tbsb: $\operatorname{Tzz}(2 \mathrm{~A})-\operatorname{Tzz}(2 \mathrm{~B})>0(t=1 \%$ e $p=0,002)$.

Outras diferenças foram testadas, mas não foi possível rejeitar a hipótese de igualdade entre as médias. Dentre elas destacamos:

1. a existência de efeito indutivo sobre Tbsb: $|\operatorname{Tbsb}(2 \mathrm{~B})-\operatorname{Tbsb}(2 \mathrm{~A})|=0(p=0,165)$;

2. a persistência de efeito indutivo para os questionários do tipo $2:|\operatorname{Tbh}(2 \mathrm{~A})-\operatorname{Tbh}(2 \mathrm{~B})|=0$ $(p=0,105)$; e

3. a existência de divergência entre os efeitos sofridos por Tbh induzidos pelo primeiro evento apresentado: $|\operatorname{Tzz}(2 \mathrm{~A})-\operatorname{Tbh}(2 \mathrm{~A})|-|\operatorname{Tzz}(1 \mathrm{~A})-\operatorname{Tbh}(1 \mathrm{~A})|=0(p=0,122)$ e $\mid \operatorname{Tbsb}(2 \mathrm{~B})-$ $\operatorname{Tbh}(2 \mathrm{~B})|-| \operatorname{Tbsb}(1 \mathrm{~B})-\operatorname{Tbh}(1 \mathrm{~B}) \mid=0(p=0,499)$.

Observamos que todos os testes para os quais não foi possível rejeitar a hipótese de igualdade entre as médias envolveram observações oriundas do segundo experimento, cujo maior número de perguntas pode ampliar a ocorrência de ruídos. Isso porque, quanto mais extenso ou repetitivo o questionário, maiores as chances de os respondentes se cansarem, reduzindo a atenção dada à leitura das perguntas.

Vamos apresentar agora os resultados dos experimentos para as três turmas, separadamente. A Tabela 6 apresenta os resultados para a turma 1.

Para a primeira turma de respondentes, podemos observar que, embora mais amenas, as diferenças entre os valores médios acompanham quase perfeitamente os parâmetros estabelecidos na análise agregada dos dados. Aqui, novamente corroboramos a $\mathrm{CIH}$ na presença de parâmetros comparativos, verificando ainda, com o segundo experimento, o efeito dessa hipótese sobre Tzz e Tbsb. Novamente os impactos comparativos parecem ser menores quanto maior o grau de competência dos indivíduos sobre o evento em questão. 0 segundo experimento mostra que a memória comparativa acompanha 0 viés estabelecido pelo primeiro parâmetro apresentado.

A única diferença com resultados obtidos na análise agregada que conseguimos constatar aqui, refere-se à diferença entre o valor atribuído ao primeiro e ao último evento apresentados nos questionários quando do tipo 1, ou do tipo 2. Embora para os questionários iniciados pelo evento Tbsb, essa diferença permaneça menor quanto maior o número de parâmetros comparativos apresentados, 0 
Tabela 6: Resultados dos Experimentos 1 e 2 - Primeira Turma

\begin{tabular}{|c|c|c|}
\hline & \multicolumn{2}{|c|}{ Experimento 1 - Dois Eventos } \\
\hline & Questionário 1A $(n=16)$ & Questionário 1B $(n=17)$ \\
\hline Primeira Pergunta & Zagzig: $\mathrm{R} \$ 41,18$ & Brasília: $\mathrm{R} \$ 66,13$ \\
\hline \multirow[t]{3}{*}{ Segunda Pergunta } & Belo Horizonte: $\mathrm{R} \$ 59,18$ & Belo Horizonte: $\mathrm{R} \$ 40,06$ \\
\hline & \multicolumn{2}{|c|}{ Experimento 2 - Três Eventos } \\
\hline & Questionário 2A $(n=18)$ & Questionário 2B $(n=18)$ \\
\hline Primeira Pergunta & Zagzig: $\mathrm{R} \$ 37,11$ & Brasília: $\mathrm{R} \$ 64,44$ \\
\hline Segunda Pergunta & Brasília: $\mathrm{R} \$ 71,17$ & Zagzig: $\mathrm{R} \$ 24,06$ \\
\hline Terceira Pergunta & Belo Horizonte: R\$ 62,06 & Belo Horizonte: R\$ 49,39 \\
\hline
\end{tabular}

mesmo não ocorre para os questionários tipo $\mathrm{A}$ - com Tzz como primeiro evento. Ademais, ressaltamos que, apesar dos níveis de significância requeridos serem mais altos, todos os testes U de Mann-Whitney apresentaram os mesmos resultados qualitativos encontrados para o total das turmas.

Tabela 7: Resultados dos Experimentos 1 e 2 - Segunda Turma

\begin{tabular}{|c|c|c|}
\hline & \multicolumn{2}{|c|}{ Experimento 1 - Dois Eventos } \\
\hline & Questionário 1A $(n=20)$ & Questionário 1B $(n=21)$ \\
\hline Primeira Pergunta & Zagzig: $\mathrm{R} \$ 45,05$ & Brasília: $\mathrm{R} \$ 54,55$ \\
\hline \multirow[t]{3}{*}{ Segunda Pergunta } & Belo Horizonte: R\$ 57,10 & Belo Horizonte: $\mathrm{R} \$ 44,10$ \\
\hline & \multicolumn{2}{|c|}{ Experimento 2 - Três Eventos } \\
\hline & Questionário 2A $(n=17)$ & Questionário 2B $(n=20)$ \\
\hline Primeira Pergunta & Zagzig: $\mathrm{R} \$ 42,60$ & Brasília: R\$ 65,29 \\
\hline Segunda Pergunta & Brasília: R\$ 63,15 & Zagzig: $\mathrm{R} \$ 35,24$ \\
\hline Terceira Pergunta & Belo Horizonte: R\$ 47,85 & Belo Horizonte: R\$ 55,35 \\
\hline
\end{tabular}

Na segunda turma, quase todos os resultados se repetem, embora de forma mais amena que para o total das observações. Aqui, encontramos uma inversão no parâmetro predominante para determinação do viés sobre Tbh nos questionários tipo 2 . Contudo, se reproduz o resultado onde a inserção de mais uma pergunta num questionário tipo 1 , transformando-o em um questionário tipo 2 , leva a uma redução do impacto do parâmetro inicial sobre o evento de familiaridade moderada - Tbh. Além disso, destacamos que nenhuma das diferenças entre os preços médios foi corroborada pelo teste $\mathrm{U}$ de Mann-Whitney.

Por fim, para a terceira turma de respondentes, todos os resultados encontrados para o agregado se repetem, alguns dos quais apresentando diferenças ainda maiores, reforçando os resultados para o conjunto das turmas. Para esta turma, embora tenha sido observada uma diferença entre os valores médios atribuídos à loteria Tbh no experimento 1, ela não é estatisticamente significante. Contudo, a diferença entre os valores médios atribuídos a essa mesma loteria no experimento 2 apresenta-se maior 
e é significativa a 10\%, usando o teste de Mann-Whitney. Além disso, foi possível verificar, ainda usando o mesmo teste, um maior impacto de Tzz sobre Tbh no questionário tipo 1A, com relação ao tipo 2A.

Tabela 8: Resultados dos Experimentos 1 e 2 - Terceira Turma

\begin{tabular}{|c|c|c|}
\hline & \multicolumn{2}{|c|}{ Experimento 1 - Dois Eventos } \\
\hline & Questionário 1A $(n=25)$ & Questionário 1B $(n=24)$ \\
\hline Primeira Pergunta & Zagzig: $\mathrm{R} \$ 31,90$ & Brasília: R\$ 56,63 \\
\hline \multirow[t]{3}{*}{ Segunda Pergunta } & Belo Horizonte: $\mathrm{R} \$ 48,08$ & Belo Horizonte: $\mathrm{R} \$ 42,50$ \\
\hline & \multicolumn{2}{|c|}{ Experimento 2 - Três Eventos } \\
\hline & Questionário 2A $(n=20)$ & Questionário 2B $(n=19)$ \\
\hline Primeira Pergunta & Zagzig: $\mathrm{R} \$ 54,95$ & Brasília: R\$ 55,97 \\
\hline Segunda Pergunta & Brasília: R\$ 70,85 & Zagzig: R\$ 22,00 \\
\hline Terceira Pergunta & Belo Horizonte: $R \$ 65,00$ & Belo Horizonte: $R \$ 45,79$ \\
\hline
\end{tabular}

\section{CONCLUSÃO}

O presente trabalho realizou uma breve discussão acerca dos efeitos competência e comparação, a qual motivou a apresentação de dois experimentos, objeto principal do trabalho. Os experimentos investigam o efeito comparativo nas escolhas individuais.

Mais especificamente, aprofundamos o estudo da hipótese de ignorância comparada de Fox e Tversky (1995). Para isso, replicamos um dos experimentos realizados por Fox e Weber (2002) (experimento 1) e, em seguida, expandimos este experimento para uma situação mais abrangente (experimento 2), onde se buscou investigar o comportamento da memória comparativa dos agentes e os efeitos da comparação entre loterias dentro do processo decisório individual.

No total, quatro tipos de questionários foram elaborados, dois para cada experimento. Nestes, os respondentes deveriam estabelecer seus preços de reserva para loterias que premiariam com $R \$ 100$ a ocorrência de determinada temperatura em uma dentre três localidades distintas, a saber: Brasília (Tbsb) - evento de familiaridade supostamente alta; Belo Horizonte (Tbh) - evento de familiaridade supostamente moderada; e Zagazig, no Egito, (Tzz) - evento de familiaridade supostamente baixa. Para o experimento 1, dois tipos de questionários foram utilizados. No primeiro (1A), era oferecida aos alunos uma loteria que contemplava o evento Tzz, seguida da loteria Tbh; enquanto no segundo (1B) eram oferecidas as loterias Tbsb e Tbh, respectivamente. Para o segundo experimento, os questionários (2A e 2B) ofereciam as três loterias sob as seguintes ordenações: $2 \mathrm{~A}$ ) Tzz, Tbsb, Tbh; e 2B) Tbsb, Tzz, Tbh.

Seguindo Fox e Weber (2002), para cada uma das cidades, os indivíduos precificaram loterias complementares - temperatura maior ou igual, e temperatura menor que - e, para evitar a ocorrência de vieses gerados por crenças generalizadas sobre a ocorrência de determinada temperatura, a análise dos resultados foi realizada a partir da comparação entre as médias da soma dos preços atribuídos por cada agente a cada par de loterias complementares. O teste não-paramétrico de diferenciação de médias de Wilcoxon, Mann e Whitney, conhecido como teste U de Mann-Whitney, foi utilizado para verificar a siginificância estatística dos resultados.

A análise realizada sobre o experimento 1 nos permitiu corroborar a hipótese de ignorância comparada. o comportamento comparativo observado acompanhou o padrão dos resultados encontrados 
por Fox e Weber (2002), que mostraram ser possível induzir os indivíduos a se acharem mais ou menos competentes a respeito de um determinado evento, por meio da provisão de um parâmetro comparativo adequado.

Assim, se expusermos o indivíduo primeiro a um evento sobre o qual ele se considera bastante conhecedor, ele tenderá a subestimar seu nível de conhecimento a respeito de eventos sobre os quais se sinta menos competente. Analogamente, se o expusermos a um evento sobre o qual ele se considera ignorante, ele tenderá a superestimar seu conhecimento acerca de eventos para os quais se considere mais competente.

Fox e Tversky (1995, p. 600) levantam a possibilidade de que os julgamentos realizados em ambientes não-comparativos são mais racionais e que os indivíduos apenas sentem-se intimidados pela presença de eventos mais familiares ou de indivíduos mais competentes. Tal ideia é corroborada pelos resultados do experimento 2 , onde os preços atribuídos às loterias acompanham o nível de competência julgada dos agentes sobre os eventos precificados - preço(Tbsb) $>$ preço(Tbh) $>$ preço(Tzz) - para ambos os questionários. Além disso, se considerarmos que a resposta à pergunta realizada em primeiro lugar não sofre efeito comparativo - embora gere um parâmetro de comparação para as perguntas que se seguem - corroboramos a ideia de que o estabelecimento de parâmetros comparativos tem o poder de ampliar a aversão à ambiguidade, onde esta aversão apresenta-se tão maior quanto menor for a familiaridade do evento para o agente (Fox e Tversky, 1995, Fox e Weber, 2002, Chow e Sarin, 2001).

Outro comportamento importante verificado neste estudo indica que a percepção de conhecimento será menos vulnerável a efeitos de comparação quanto mais alto for o grau de familiaridade do evento. Assim, para eventos sobre os quais o indivíduo se considera certo (100\% de chance de sucesso), não seria possível distorcer este sentimento. Contudo, seria fácil fazê-lo crer ser mais competente sobre um evento que lhe fosse mediana ou vagamente familiar.

Por fim, os resultados levam a crer que o primeiro parâmetro comparativo apresentado possui um maior poder indutivo e que a inserção de mais perguntas/decisões pode atenuar o efeito comparação. Porém, o efeito induzido pela primeira pergunta é robusto o suficiente para influenciar a resposta da terceira pergunta.

Para este último comportamento observado, vislumbramos duas explicações possíveis. Primeiro, podemos considerar a existência de uma inércia comparativa, segundo a qual, uma vez que o agente estabeleça um parâmetro de comparação, ele terá dificuldade de estabelecer novos, dentro de um mesmo contexto decisório. Neste caso, a redução no efeito indutivo sobre a última questão pode advir não de um choque entre parâmetros opostos, mas do simples afastamento entre ela e o parâmetro de comparação estabelecido - evento abordado na primeira questão -, afastamento este que teria o poder de reduzir a memória comparativa do indivíduo. A segunda explicação envolve um raciocínio mais minucioso. Considere que, ao deparar-se com uma loteria inicial (A), o agente ainda não possui uma base comparativa. Nessa situação, ele pondera e constroi uma estrutura de crenças a respeito de seu próprio nível de conhecimento. Com base nessas crenças, ele valora a loteria A. Agora, suponha que o agente é exposto a uma segunda loteria (B). Para avaliar B, utiliza as crenças que formou a partir de A - hipótese de ignorância comparada.

Finalmente, ao ser confrontado com uma terceira loteria (C), o agente poderia:

1) manter sua estrutura de crenças, a qual toma por base a loteria $A$;

2) modificar sua estrutura de crenças, comparando a loteria C com a B;

3) formar crenças baseadas nas loterias A e B e avaliar a loteria C com base nelas; e

4) desprezar as loterias anteriores e avaliar a loteria C, sem realizar comparações.

Embora essa decisão não seja claramente ponderada, a reação do agente refletirá um balanceamento dos benefícios e custos atrelados a cada resultado. Assim, se ele mantém sua estrutura de crenças inicial 
- caso 1 - e perde, ele incorrerá no custo da perda. Contudo, se ele opta por abrir mão dessa estrutura - seguindo os casos 2, 3 ou 4 - e perde, ele não apenas sofrerá o custo da perda, mas também agregará a ela o custo do arrependimento por ter modificado sua estrutura de crenças inicial. Ou seja, o agente apoia suas decisões no primeiro parâmetro comparativo apresentado por antecipar os custos associados à perda caso ele mantenha suas crenças iniciais e caso as altere.

Deste modo, podemos inferir que se o indivíduo apostou inicialmente na loteria $\mathrm{A}$, é porque acredita na possibilidade de ganho de A. Então, o sentimento de perder apostando em A é menos incômodo do que o sentimento de ver A ganhar, uma vez que ele tenha desistido de A em favor de B.

Bernasconi e Loomes (1992) apresentaram um experimento cujo resultado observamos um padrão comportamental que corrobora essa ideia. Os autores aplicaram um experimento de Ellsberg com urnas de três cores, onde é usada uma urna com 30 bolas vermelhas, cor não ambígua, e 60 bolas pretas ou amarelas, cores ambíguas, pois estão em proporção desconhecida. Apostas nas cores, similares ao experimento com duas cores, são oferecidas aos participantes. Em uma primeira rodada, aproximadamente $50 \%$ dos indivíduos preferiram apostar na cor não ambígua e desses, $90 \%$ se recusaram a trocar sua aposta de $£ 10,00$ na cor não ambígua por uma aposta de $£ 12,00$ numa das cores ambíguas. Contudo, na segunda rodada, quando avisados de que poderiam apostar em duas cores, aqueles que haviam apostado numa das cores ambíguas, preferiram, em geral, apostar na combinação não ambígua de cores. Enquanto os que optaram pela cor não ambígua deram preferência a sua simples associação, gerando uma preferência por combinações ambíguas. Esse resultado pode ser interpretado como a existência de um alto grau de resistência dos indivíduos a abandonarem suas escolhas iniciais, mesmo quando mantê-las os levaria a situações de incerteza knightiana.

Assim, o presente trabalho corrobora a ideia de que, provido dos parâmetros de comparação adequados, um indivíduo pode ser levado a crer-se mais ou menos conhecedor acerca de um mesmo evento. Além disso, indica que, na presença de mais de um parâmetro comparativo, cujos fluxos indutivos os levem em direções opostas, os agentes tendem a seguir o primeiro parâmetro apresentado.

\section{BIBLIOGRAFIA}

Allais, M. (1953). Le comportement de l'homme rationnel devant le risque: Critique des postulats et axiomes de l'école américaine. Econometrica, 21(4):503-546.

Becker, S. W. \& Brownson, F. O. (1964). What price ambiguity?Or the role of ambiguity in decisionmaking. Journal of Political Economy, 72:62-73.

Bernasconi, M. \& Loomes, G. (1992). Failures of the reduction principle in an Ellsberg-type problem. Theory and Decision, 32:77-100.

Camerer, C. F. \& Hogarth, R. (1999). The effects of financial incentives in experiments: A review and capital-labor-production framework. Journal of Risk and Uncertainty, 3:7-42.

Camerer, C. F. \& Weber, M. (1992). Recent developments in modeling preferences: Uncertainty and ambiguity. Journal of Risk and Uncertainty, 5:325-370.

Chow, C. C. \& Sarin, R. K. (2001). Comparative ignorance and the Ellsberg paradox. Journal of Risk and Uncertainty, 2:129-139.

de Lara Resende, J. \& Wu, G. (2010). Competence effects for choices involving gains and losses. Journal of Risk and Uncertainty, 40:109-132.

Dellavigna, S. (2009). Psychology and economics: Evidence from the Field. Journal of Economic Literature, 47(2):315-372. 
Ellsberg, D. (1961). Risk, ambiguity and the Savage axioms. Quarterly Journal of Economics, 75:643-669.

Fox, C. R. \& Tversky, A. (1995). Ambiguity aversion and comparative ignorance. Quarterly Journal of Economics, 110:585-603.

Fox, C. R. \& Tversky, A. (1998). A belief-based account of decision under uncertainty. Management Science, 44:879-895.

Fox, C. R. \& Weber, M. (2002). Ambiguity aversion, comparative ignorance and decision context. Organizational Behavior and Human Decision Processes, 88:476-498.

Heath, C. \& Tversky, A. (1991). Preference and belief: Ambiguity and competence in choice under uncertainty. Journal of Risk and Uncertainty, 4:5-28.

Hertwig, R. \& Ortmann, A. (2001). Experimental practices in economics: A methodological challenge for psychologists? Behavioral and Brain Sciences, 24:383-403.

Keynes, J. M. (1921). A treatise on probability. London, Macmillan.

Knight, F. (1921). Risk, uncertainty, and profit. New York, Hougthon Mifflin Company.

Savage, L. (1954). The Foundations of Statistics. New york, Wiley.

von Neumann, J. \& Morgentern, O. (1944). Theory of Games and Economic Behavior. Princeton University Press, Princeton University. 